\title{
Expression of ALDH1A1 and CD133 is associated with the prognosis and effect of different chemotherapeutic regimens in gastric cancer
}

\author{
WAN-TING LIU, WEN-BO LIU, MIN GAO, YI-YIN ZHANG and KANG-SHENG GU
}

Department of Oncology, The First Affiliated Hospital of Anhui Medical University, Hefei, Anhui 230022, P.R. China

Received February 2, 2019; Accepted July 26, 2019

DOI: $10.3892 / 01.2019 .10798$

\begin{abstract}
Gastric cancer (GC) is one of the most common malignant tumors worldwide. Previous studies have reported that aldehyde dehydrogenase-1A1 (ALDH1A1) and cluster of differentiation (CD)-133 are considered to be cancer stem cell markers in GCs. The present study immunohistochemically examined the distribution and expression of two tumor stem cell markers, CD133 and ALDH1A1, in both primary tumors and para-tumor tissues. In 91 cases with stage III, 57 (62\%) were positive for ALDH1A1 and 60 (66\%) were positive for CD133. ALDH1A1 was detected in para-tumors and cancerous tissues of the stomach, and the immunoreactivity of the tumors was stronger than that in para-tumor tissues. CD133 was only detected in tumors. The expression of ALDH1A1 was significantly associated with advanced $\mathrm{T} / \mathrm{N}$ stage ( $\mathrm{T}$ stage, $\mathrm{P}=0.012$; $\mathrm{N}$ stage, $\mathrm{P}=0.023)$ and poor differentiation $(\mathrm{P}=0.020)$, while $\mathrm{CD} 133$ was associated with advanced $\mathrm{T}$ stage $(\mathrm{P}=0.007)$. Univariate and multivariate Cox proportional hazards analysis revealed that tumor stage, CD133 expression, vascular invasion and sex were independent predictors of disease-free survival (DFS) time, and tumor size, vascular invasion and sex were independent predictors of overall survival (OS) time in patients with GC. Patients with $\mathrm{CD} 133^{+} \mathrm{GC}$ had poorer DFS ( $\left.\mathrm{P}=0.042\right)$, while $\mathrm{ALDH} 1 \mathrm{~A} 1^{+} \mathrm{GC}$ was not associated with poorer DFS. In regard to chemotherapy, improvements in survival were not observed after the addition of taxane compared with two-drug therapy. However, the subgroup analysis indicated that in the

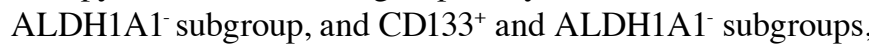
an increased $\mathrm{OS}$ was observed in two-drug therapy $(\mathrm{P}=0.043)$. The results of the present study indicate that ALDH1A1 and CD133 may play an important role in tumor invasion, metastasis and prognosis, and ALDH1A1' expression does not
\end{abstract}

Correspondence to: Professor Kang-Sheng Gu, Department of Oncology, The First Affiliated Hospital of Anhui Medical University, 218 Jixi Road, Hefei, Anhui 230032, P.R. China E-mail: 13805692145@163.com

Key words: aldehyde dehydrogenase-1A1, cluster of differentiation-133, stomach neoplasm, prognosis, adjuvant chemotherapy benefit the taxane-based triple chemotherapeutic regimen in patients with GC.

\section{Introduction}

Gastric cancer (GC) is one of the most common malignancies worldwide (1). Global cancer statistics have revealed that approximately 951,600 new cases of GC and 723,100 deaths were reported in 2012 (1). Cancer stem cells (CSCs) have been suggested to be associated with tumor initiation, drug resistance, invasion, metastasis and tumor recurrence, and therefore may play an important role in cancer-associated mortality (2). CSCs are also known as tumor-initiating cells, with self-renewal and proliferative characteristics (3). By studying different CSCs, one can select various individualized chemotherapies and improve the survival rate of patients with GC. In some studies $(3,4), \mathrm{CSCs}$, such as cluster of differentiation (CD)-24, CD26, CD44, CD90, CD133 and aldehyde dehydrogenase-1A1 (ALDH1A1), have been detected in GC, which were expressed with high carcinogenicity in animal experiments.

CD133 is a glycoprotein with five transmembrane domains (5). Initially, CD133 was considered to be a surface marker for hematopoietic stem and progenitor cells (6). An increasing number of studies have reported that CD133 is also a marker for colorectal (7), liver (8), prostate (9) and lung cancer (10), and it is often used to label and isolate stem cells. Approximately half of human GC samples express $\mathrm{CD}_{133^{+}}$, and CD133 is associated with the development of tumors. A high expression of CD133 always leads to shorter survival $(11,12)$.

ALDH1A1 is a type of intracellular enzyme with self-renewal and multi-directional differentiation (13). It plays a part in the differentiation of CSCs by stimulating the formation of retinoic acid, and participates in the detoxification, differentiation and drug resistance of cells (13). ALDH1A1 has already been reported in colorectal cancer, lung cancer, breast cancer and other types of tumors, however to the best of our knowledge, a limited number of studies have reported the role of ALDH1A1 in GC. A number of studies have reported that ALDH1 overexpression is associated with low survival in malignant tumors $(14,15)$.

D2 gastrectomy has become the standard surgical treatment for curable GC globally (16). Complete resection of the tumor is critical; however, the tumor may recur even if it is completely 
resected. In terms of further treatment after gastrectomy, two landmark phase III clinical trials in Asia, the adjuvant chemotherapy ACTS-GC and the CLASSIC trials $(17,18)$, reported that both monotherapy and combination chemotherapy were associated with a reduced risk of death in GC compared with surgery alone. In addition, standard postoperative adjuvant chemotherapy was established. Subgroup analysis in the ACTS-GC trial revealed the important benefits of S-1 monotherapy in patients with early stage disease. By contrast, patients with late stage did not exhibit any benefits and they required a more efficient treatment. In the CLASSIC trial, despite the results demonstrating that the XELOX therapy was beneficial for all disease stages (II, IIIA or IIIB), there was still no standard for adjuvant chemotherapy for the patients who were excluded.

In the present study, the response of different chemotherapies under positive or negative expression of CD133 and ALDH1A1 were investigated in order to examine the value of CD133 and ALDH1A1 in the treatment of GC.

\section{Materials and methods}

Patients and tissue samples. The objective was to evaluate the association between different adjuvant chemotherapies and the expression of CSCs in patients with stage III, GC who had undergone curative resection. The present study was approved by the Institutional Review Board and Human Ethics Committee of The First Affiliated Hospital of Anhui Medical University. All the patients signed written consent for the use of their samples for research purposes. Cancerous and para-tumor tissues were obtained after surgery from the Department of Pathology, The First Affiliated Hospital of Anhui Medical University. Hematoxylin \& eosin (H\&E)-stained slides of gastric cancer and normal gastric mucosa were obtained from the same department.

In the present retrospective analysis, a total of 91 patients were enrolled, including 69 males and 22 females. Patient age ranged from 32 to 76 years, and the median age was 53 years. The gastrectomies were performed between January 2012 and December 2017. The inclusion criteria were: i) Age between 18 and 80 years; ii) Eastern Cooperation Oncology Group score between 0 and 2 (19); iii) R0 gastrectomy with D2 or more extensive lymphadenectomy; iv) pathological stage confirmed as stage III (according to The 8th edition of the AJCC Cancer Staging Manual (20), resulting in a slightly different stage III population between this study and other ones); v) no radiotherapy and/or immunotherapy; vi) complete clinical data; and vii) baseline examination met the requirements of chemotherapy (adequate hematologic function, hepatic function, and renal function).

Immunohistochemical staining and assessment. Immunohistochemistry was carried out according to a procedure previously reported $(21,22)$. Paraffin-embedded blocks were cut into $4-\mu \mathrm{m}$ sections. Firstly, samples were deparaffinized twice in xylene for $10 \mathrm{~min}$, and then dehydrated in a graded ethanol series $(100 \%$ ethanol twice, then 95 and $75 \%$ ethanol). Secondly, antigen retrieval in $0.01 \mathrm{M}$ sodium citrate buffer ( $\mathrm{pH}$ 6.0) was performed at a high temperature and high pressure using a pressure cooker, then endogenous peroxidase activity was quenched by $10 \mathrm{~min}$ incubation in
$3 \% \mathrm{H}_{2} \mathrm{O}_{2}$ solution after cooling to room temperature, followed by 3 washes for 3 min each with PBS ( $\mathrm{pH} \mathrm{7.4).} \mathrm{The} \mathrm{sections}$ were incubated with $3 \% \mathrm{H}_{2} \mathrm{O}_{2}$ solution for $10 \mathrm{~min}$ at room temperature to inhibit endogenous peroxidase activity. After 30 min of blocking in $10 \%$ goat serum (cat. no. SP KIT-B1; Maxim Biotech, Inc.) at room temperature, the sections were incubated with primary antibodies: anti-ALDH1A1 (Abcam; cat. no. ab52492; dilution 1:200) and anti-CD133 (Abcam; cat. no. ab222782; dilution 1:500), in a refrigerator at $4^{\circ} \mathrm{C}$ overnight. The next day, the sections were rinsed three times for 3 min each with PBS, followed by incubating array slides with anti-mouse/rabbit secondary antibodies [MaxvisionTM3 HRP-Polymer (Mouse/Rabbit) IHC kit, Maxim Biotech, Inc.; cat. no. KIT-5220] for $20 \mathrm{~min}$ at $37^{\circ} \mathrm{C}$. Samples were then stained using a DAB kit for $10 \mathrm{~min}$ at room temperature, and hematoxylin for $1 \mathrm{~min}$ at room temperature before dehydration and transparency of slides; finally, samples were mounted on slides. The staining was observed using a light microscope (magnification, x100, x200 and x400).

A total of 10 high-power fields were randomly selected for each slice, and 100 tumor cells were counted in each field of view. The assessments of the two CSCs were: If the number of positive cells was $<5,5-24,25-49,50-75$ or $>75 \%$, the slice was marked as $0,1,2,3$ or 4 points, respectively. Judging the intensity of the two indicators: The range of intensities was from 0 to 3, for colorless, yellow, brown and tan, respectively. Finally, the positive expression rate score and the intensity score were multiplied. The present study considered the scores $0,2-4,5-8$ and 9-12, for CD133 and ALDH1A1, equal to negative, weakly positive, positive and strongly positive, respectively. Immunohistochemical studies for ALDH1A1 and CD133 were performed and scored semiquantitatively by two pathologists based on the percent and intensity of positive cells as previously described $(21,22)$. The assessments of slices were conducted using the double-blind method.

Treatment and assessment. All patients were enrolled in postoperative chemotherapy, under the combination of two [platinum + 5-fluorouracil (5-FU)] or three drug (taxane + platinum $+5-\mathrm{FU}$ ) treatments. Patients who were treated with two-drug combinations received XELOX chemotherapy (capecitabine $1,000 \mathrm{mg} / \mathrm{m}^{2}$ PO BID on days 1-14 and oxaliplatin $130 \mathrm{mg} / \mathrm{m}^{2} \mathrm{IV}$ on day 1 , cycled every 21 days for 6-8 cycles) with SOX (S-1 $80 \mathrm{mg} / \mathrm{m}^{2}$ PO BID on days 1-14 and oxaliplatin $130 \mathrm{mg} / \mathrm{m}^{2} \mathrm{IV}$ on day 1 , cycled every 21 days for 6-8 cycles) chemotherapy, or 12 cycles of mFOLFOX6 chemotherapy (oxaliplatin $85 \mathrm{mg} / \mathrm{m}^{2} \mathrm{IV}$ on day 1 and leucovorin $400 \mathrm{mg} / \mathrm{m}^{2} \mathrm{IV}$ on day $1,5-\mathrm{FU} 400 \mathrm{mg} / \mathrm{m}^{2} \mathrm{IV}$ bolus on day 1 , then $1,200 \mathrm{mg} / \mathrm{m}^{2} / \mathrm{d}$ IV continuous infusion on days 1 to 2 , repeated every 2 weeks). Patients treated with three-drug combinations received DCF (docetaxel $75 \mathrm{mg} / \mathrm{m}^{2} \mathrm{IV}$ on day 1 and cisplatin $75 \mathrm{mg} / \mathrm{m}^{2}$ IV on day $1,5-F U ~ 750 \mathrm{mg} / \mathrm{m}^{2} \mathrm{IV}$ on days 1 to 5 ) or $\mathrm{mDCF}$ (docetaxel $40 \mathrm{mg} / \mathrm{m}^{2} \mathrm{IV}$ on day 1 , leucovorin $400 \mathrm{mg} / \mathrm{m}^{2} \mathrm{IV}$ on day $1,5-\mathrm{FU} 400 \mathrm{mg} / \mathrm{m}^{2} \mathrm{IV}$ on day $1,5-\mathrm{FU} 1,200 \mathrm{mg} / \mathrm{m}^{2} \mathrm{IV}$ continuous infusion over $24 \mathrm{~h}$ on day 1 and 2, cycled every 14 days). The present study adhered to the principles of 'NCCN Clinical Practice Guidelines on Oncology, Gastric Cancer' (23). Before chemotherapy, the patient's hematopoiesis, liver and kidney functions were assessed and recorded. Tumor assessment was conducted using 
tumor markers, computed tomography or magnetic resonance imaging, and gastroscopy every 2 cycles during chemotherapy. According to the results it was unknown whether the tumor recurred or metastasized. Adverse events were evaluated according to the National Cancer Institute's Common Terminology Criteria for Adverse Events (version 3.0) (24). If patients had serious hematologic toxic effects, neurological adverse events, intolerant digestive tract reactions or severe hand-foot syndrome, dose reductions were allowed. In cases of reducing the dose, patients that could not tolerate chemotherapy were allowed to interrupt and/or withdraw from the study.

Follow-up. The follow-up of patients was mainly conducted by telephone and by using the hospital medical records. Follow-up was performed every 3 months from the first to second year after gastrectomy, and every 6 months in the last 2-5 years. The primary endpoint was disease-free survival (DFS) and overall survival (OS). DFS was defined as the time from the date of gastrectomy to the date of the recurrence of the original GC or end of the follow-up. OS was defined as the date from gastrectomy to the date of death or the last follow-up.

Statistical analysis. The statistical analyses were conducted using IBM SPSS version 25.0 (IBM Corp.). Data are expressed as the mean \pm standard deviation. Comparison of the categorical data was performed by the $\chi^{2}$ test. Fisher's exact test with a residual analysis as a post-hoc test (only in the group of the association between $\mathrm{T} / \mathrm{N}$ stage and expression of ALDH1A1 and CD133) was performed. The Spearman rank correlation test was used for correlation analysis. The Kaplan-Meier survival curves were constructed to estimate the cumulative survival. The survival rate between two chemotherapy groups was compared by a two-sided log-rank test. The statistically significant variables with $\mathrm{P}<0.05$ in the univariate analysis were included in the Cox proportional hazards model. The survival curves were drawn using GraphPad Prism 7 (GraphPad Software, Inc.). Immunohistochemical staining and assessment were repeated 3 times.

\section{Results}

Association between ALDH1A1 and CD133 with clinicopathological features. According to the immunohistochemistry results, 57/91 (62\%) were positive for ALDH1A1 and 60/91 $(66 \%)$ were positive for CD133. Expression of ALDH1A1 was mainly observed in the cytoplasm of GC cells, which were stained yellow, brown and tan. Not only were the tumor cells stained but they were also strongly active in normal gastric mucosa cells, especially parietal cells. CD133 was detected in the cytomembrane, but not in normal gastric epithelial and stromal cells (Figs. 1 and 2). The results of the univariate analysis indicated that $\mathrm{T}$ stage $(\mathrm{P}=0.012), \mathrm{N}$ stage $(\mathrm{P}=0.023)$, and histological type $(\mathrm{P}=0.020)$ were significantly associated with positive ALDH1A1 expression. After analysis by the post-hoc test, ALDH1A1- tended to indicate T2 or N1 stage, while $\mathrm{ALDH} 1 \mathrm{~A} 1^{+}$tended to indicate $\mathrm{T} 4 \mathrm{~b}$ stage. In addition, there was an interaction between $\mathrm{T}$ stage $(\mathrm{P}=0.007)$ and $\mathrm{CD} 133^{+}$ (Table I). After analysis via post-hoc tests, CD133- tended to indicate $\mathrm{T} 2$ stage, and $\mathrm{CD} 133^{+}$tended to indicate $\mathrm{T} 4 \mathrm{a}$ stage.
It was also found that the level of expression was associated with the stages as determined by Spearman's rank correlation coefficient, i.e., the higher the ALDH1A1 expression, the later the $\mathrm{T}$ stage $(\mathrm{r}=0.224 ; \mathrm{P}=0.011)$. However, neither the $\mathrm{N}$ stage nor the level of CD133 expression reflected this association.

Effect of tumor ALDHIAI and CD133 protein level on prognosis. Kaplan-Meier analysis proved that a significant difference for DFS was observed between positive and negative CD133 expression ( $\mathrm{P}=0.042)$; however, this did not apply for the OS curve. High expression of CD133 was associated with poor DFS. By contrast, the DFS curve of ALDH1A1 ${ }^{+}$ expression did not indicate a significant difference compared with the ALDH1A1 ${ }^{-}$expression. The same applied for the OS curve. High expression of ALDH1A1 was not associated with better DFS, and no statistical significance was revealed after the log-rank test (Fig. 3A-D).

CD133 and ALDH1A1 co-expression, which refers to the combined expression of two markers, was also associated with DFS. Patients with $\mathrm{CD}_{133^{+}}$and $\mathrm{ALDH} 1 \mathrm{~A} 1^{-}$expression exhibited a significant decrease in $\mathrm{DFS}(\mathrm{P}=0.034)$; by contrast, patients with $\mathrm{CD}^{-} 33^{-}$and $\mathrm{ALDH} 1 \mathrm{~A} 1^{-}$expression exhibited a slow decrease (Fig. 3E and F). A poor positive correlation was revealed between the degree of ALDH1A1 and CD133 expression by using Spearman's rank correlation coefficient ( $\mathrm{rs}=0.240 ; \mathrm{P}=0.022$ ).

Association between chemotherapies and clinical characteristics. The endpoint of the follow-up date was September 31, 2018, with a median follow-up of 27 months. The present study detected the association between clinical characteristics and different chemotherapies. All patients had complete clinical data and successful follow-ups. There was a significant difference between patient age and therapy $(\mathrm{P}<0.05)$. The patients who were 60 years of age were predominantly treated with a combination of two drugs. The remaining general data showed no statistical significance $(\mathrm{P}>0.05)$. A total of 59 patients $(64.8 \%)$ were treated with two drugs, and 32 patients $(35.2 \%)$ were treated with three drugs. The three-drug group included the addition of taxane when compared with two-drug group. The specific treatment regimen was as follows: 36 patients $(39.6 \%)$ received XELOX, 19 patients $(20.9 \%)$ received SOX, 4 patients received mFOLFOX6 (4.4\%), 11 patients $(12.1 \%)$ received DCF and 21 patients $(23.1 \%$ ) received $\mathrm{mDCF}$ (data not shown).

Effect of different chemotherapies on prognosis. The DFS and OS rates at 1,2 and 3 years were calculated using the $\chi^{2}$ test. The results revealed that there were no significant differences between the 1-year DFS, 2-year DFS and the OS for the two groups (1-year DFS, $\mathrm{P}=0.645$; 2-year DFS, $\mathrm{P}=0.537$; 1 -year OS, $\mathrm{P}=0.357$; 2-year OS, $\mathrm{P}=0.521$; 3-year OS, $\mathrm{P}=0.833$ ). Although the 3 -year DFS of the two-drug group was $\sim 10 \%$ higher than the three-drug group, statistical significance was not indicated after the $\chi^{2}$ test $(\mathrm{P}=0.42$; Table II)

Fig. $3 \mathrm{G}$ and $\mathrm{H}$ presents DFS and OS curves for all patients with stage III disease involved in the present study. No statistically significant difference was observed. The mean DFS was 30.1 and 35.6 months in the two- and three-drug groups, respectively. The median DFS of the two-drug group was 32.0 months [95\% confidence interval (CI): 20.8-43.2], however the median DFS of the three-drug group was not 

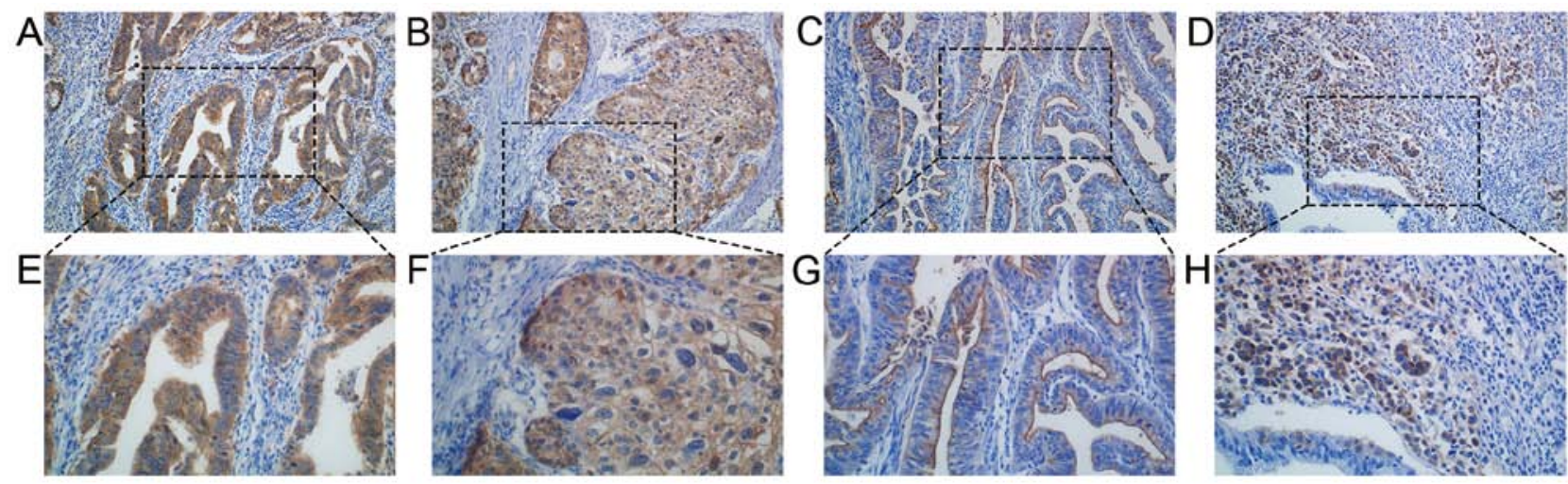

Figure 1. Immunohistochemical analysis of cancer stem cell markers in gastric cancer tissue. Expression of ALDH1A1 in (A) differentiated and (B) undifferentiated gastric cancer. Expression of CD133 in (C) differentiated and (D) undifferentiated gastric cancer. Expression of ALDH1A1 in (E) differentiated and (F) undifferentiated gastric cancer. Expression of CD133 in (G) differentiated and (H) undifferentiated gastric cancer. Magnification, x200 (A-D); magnification, x400 (E-H). L-type, (C and G); C-type, D and H. ALDH1A1, aldehyde dehydrogenase-1A1; CD133, cluster of differentiation 133; L-type, luminal expression; C-type, cytoplasmic expression.
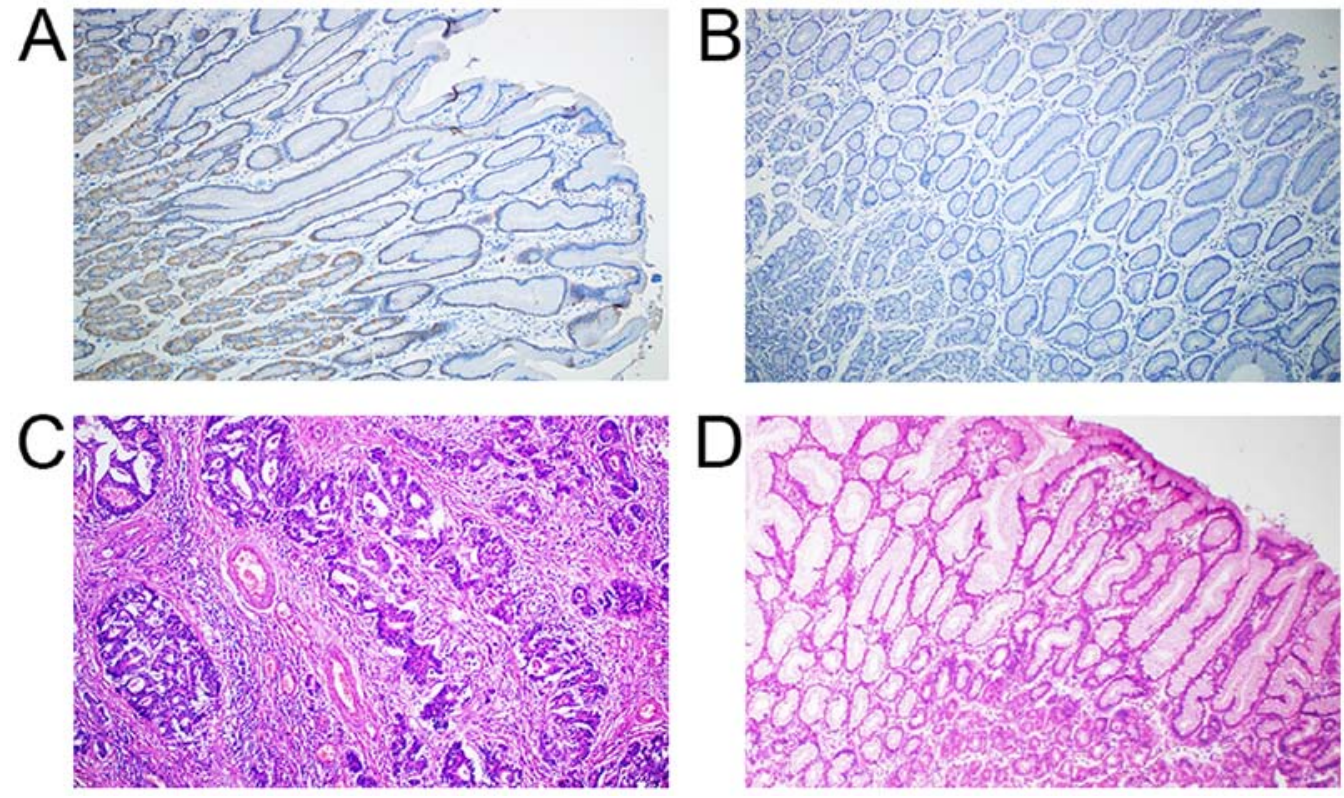

Figure 2. Immunohistochemical analysis of cancer stem cell markers in para-tumor gastric mucosa and H\&E staining in gastric cancer and normal gastric mucosa. Expression of (A) ALDH1A1 and (B) CD133 in para-tumor normal gastric mucosa. H\&E staining in (C) gastric cancer and (D) normal gastric mucosa. Magnification, x100. ALDH1A1, aldehyde dehydrogenase-1A1; CD133, cluster of differentiation 133.

reached. Statistical significance was not observed in the DFS curve $(\mathrm{P}=0.423)$. As with DFS, statistical significance was also not observed for the OS for the two groups. The mean OS was 37.9 and 39.0 months in the two- and three-drug groups, respectively. The median DFS of the two-drug group was 38.0 months (95\% CI, 32.4-43.6), and the median DFS of the three-drug group was not reached.

Multivariate Cox regression of prognosis. To evaluate the independent prognostic factors of ALDH1A1 and CD133 overexpression, univariate Kaplan-Meier survival curves for tumor size, stage, vascular invasion, and CD133 and ALDH1A1 expression were generated. Then multivariate analysis was performed using a Cox proportional hazards model in which a significant difference was observed. CD133 expression [hazard ratio (HR), 2.292; 95\% CI, 1.114-4.713; P=0.024], sex
(HR, 0.383; 95\% CI, 0.171-0.855; $\mathrm{P}=0.019$ ), stage IIIB vs. IIIA (HR, 2.746; 95\% CI, 1.306-5.773; $\mathrm{P}=0.004)$ and vascular invasion (HR, 0.627; 95\% CI, 1.003-3.494; $\mathrm{P}=0.049$ ) were independent prognostic factors for DFS after eliminating the influence of treatments. In addition, sex (HR, 0.296; 95\% CI, 0.112-0.786; $\mathrm{P}=0.015)$, tumor size $(\mathrm{HR}, 2.320$; 95\% CI, 1.125-4.784; $\mathrm{P}=0.023)$, and vascular invasion $(\mathrm{HR}, 2.516$; 95\% CI, 1.227-3.162; $\mathrm{P}=0.012$ ) were independent prognostic factors for OS after eliminating the influence of treatments (Tables III and IV).

Subgroup analysis. The present study also performed subgroup analysis of these cases as presented in Fig. 3. After Kaplan-Meier analysis, the DFS of different chemotherapies was not significantly altered in the ALDH1 $1^{-}$or ALDH1 $1^{+}$ expression subgroups. By contrast, the OS survival curve 
Table I. Association between clinical characteristics and expression of ALDH1A1 and CD133 in 91 cases of gastric cancer.

\begin{tabular}{|c|c|c|c|c|c|c|}
\hline \multirow[b]{2}{*}{ Characteristics } & \multicolumn{2}{|c|}{ ALDH1A1 } & \multirow[b]{2}{*}{ P-value } & \multicolumn{2}{|c|}{ CD133 } & \multirow[b]{2}{*}{ P-value } \\
\hline & Positive & Negative & & Positive & Negative & \\
\hline $\operatorname{Sex}(\%)$ & & & 0.368 & & & 0.794 \\
\hline Male & $45(78.9)$ & $24(70.6)$ & & $46(76.7)$ & $23(74.2)$ & \\
\hline Female & $12(21.1)$ & $10(29.4)$ & & $14(23.3)$ & $8(25.8)$ & \\
\hline Age, years $(\%)$ & & & 0.802 & & & 0.142 \\
\hline$<60$ & $32(56.1)$ & $20(58.8)$ & & $31(51.7)$ & $21(67.7)$ & \\
\hline$\geq 60$ & $25(43.9)$ & $14(41.2)$ & & $29(48.3)$ & $10(32.3)$ & \\
\hline TNM stage (\%) & & & 0.879 & & & 0.900 \\
\hline IIIA & $28(49.1)$ & $15(44.1)$ & & $28(46.7)$ & $15(48.4)$ & \\
\hline IIIB & $19(33.3)$ & $13(38.2)$ & & $22(36.7)$ & $10(32.2)$ & \\
\hline IIIC & $10(17.5)$ & $6(17.6)$ & & $10(16.7)$ & $6(19.4)$ & \\
\hline T Stage $(\%)$ & & & $0.012^{\mathrm{a}}$ & & & $0.007^{\mathrm{a}}$ \\
\hline $\mathrm{T} 2$ & 0 & $3(8.8)$ & & 0 & $3(9.7)$ & \\
\hline $\mathrm{T} 3$ & $20(35.1)$ & $17(50.0)$ & & $23(38.3)$ & $14(45.2)$ & \\
\hline $\mathrm{T} 4 \mathrm{a}$ & $31(54.4)$ & $14(41.2)$ & & $35(58.3)$ & $10(32.3)$ & \\
\hline $\mathrm{T} 4 \mathrm{~b}$ & $6(10.5)$ & 0.000 & & $2(3.3)$ & $4(12.9)$ & \\
\hline N Stage $(\%)$ & & & $0.023^{\mathrm{a}}$ & & & 0.865 \\
\hline NO & $3(5.3)$ & 0.000 & & $2(3.3)$ & $1(3.2)$ & \\
\hline $\mathrm{N} 1$ & $11(19.3)$ & 0.000 & & $9(15.0)$ & $2(6.5)$ & \\
\hline N2 & $15(26.3)$ & $12(35.3)$ & & $17(28.3)$ & $10(32.3)$ & \\
\hline N3a & $19(33.3)$ & $16(47.1)$ & & $22(36.7)$ & $13(41.9)$ & \\
\hline $\mathrm{N} 3 \mathrm{~b}$ & $9(15.8)$ & $6(17.6)$ & & $10(16.7)$ & $5(16.1)$ & \\
\hline Tumor size, $\mathrm{cm}(\%)$ & & & 0.098 & & & 0.556 \\
\hline$<5$ & $25(43.9)$ & $21(61.8)$ & & $29(48.3)$ & $17(54.8)$ & \\
\hline$\geq 5$ & $32(56.1)$ & $13(38.2)$ & & $31(51.7)$ & $14(45.2)$ & \\
\hline Histological type (\%) & & & $0.020^{\mathrm{a}}$ & & & 0.287 \\
\hline Differentiated & $12(21.1)$ & $15(44.1)$ & & $20(33.3)$ & $7(22.6)$ & \\
\hline Undifferentiated & $45(78.9)$ & $19(55.9)$ & & $40(66.7)$ & $24(77.4)$ & \\
\hline Vascular invasion $(\%)$ & & & 0.716 & & & 0.859 \\
\hline Positive & $24(42.1)$ & $13(38.2)$ & & $24(40.0)$ & $13(41.9)$ & \\
\hline Negative & $33(57.9)$ & $21(61.8)$ & & $36(60.0)$ & $18(58.1)$ & \\
\hline Nerve invasion $(\%)$ & & & 0.939 & & & 0.372 \\
\hline Positive & $18(31.6)$ & $11(32.4)$ & & $21(35.0)$ & $8(25.8)$ & \\
\hline Negative & $39(68.4)$ & $23(67.6)$ & & $39(65.0)$ & $23(74.2)$ & \\
\hline
\end{tabular}

${ }^{\mathrm{a}}<0.05$. between two variables. ALDH1A1, aldehyde dehydrogenase-1A1; CD133, cluster of differentiation 133 .

revealed that patients in the ALDH1A1- subgroup could achieve better survival time with two drugs, while patients in the $\mathrm{ALDH} 1 \mathrm{~A} 1^{+}$subgroup exhibited a longer survival time with three drugs. Statistical analyses demonstrated that two drugs were better for improving the OS of the ALDH1A1 ${ }^{-}$subgroup $(\mathrm{P}=0.043$; Fig. 4A), however the positive subgroup was not significantly different $(\mathrm{P}=0.078)$. No difference was observed in the two chemotherapies groups regardless of positive or negative CD133 expression.

Associations in the chemotherapies were also observed in the various CD133 and ALDH1A1 co-expression groups. Patients with $\mathrm{CD} 133^{+}$and $\mathrm{ALDH} 1 \mathrm{~A} 1^{-}$expression benefited more in regard to OS from the combination of two drugs
( $\mathrm{P}=0.015$; Fig. 4B). In patients with $\mathrm{CD} 133^{-}$and $\mathrm{ALDH} 1 \mathrm{~A} 1^{+}$ expression as well as $\mathrm{CD}_{133^{+}}$and $\mathrm{ALDH} 1 \mathrm{~A} 1^{+}$expression, the curve of the three-drug group was separated from the other in regard to DFS and OS, however no significant differences were observed.

The present study also separated patients who had vascular metastasis or not into two subgroups, and the results indicated whether there was metastasis in the vasculature that also had a different reaction to chemotherapies. The DFS of patients with no vascular invasion exhibited a better reaction to three-drug therapy $(\mathrm{P}=0.039)$, while there was no statistical difference in the DFS of patients with vascular invasion. The same applied for the OS in the two subgroups (data not shown). 

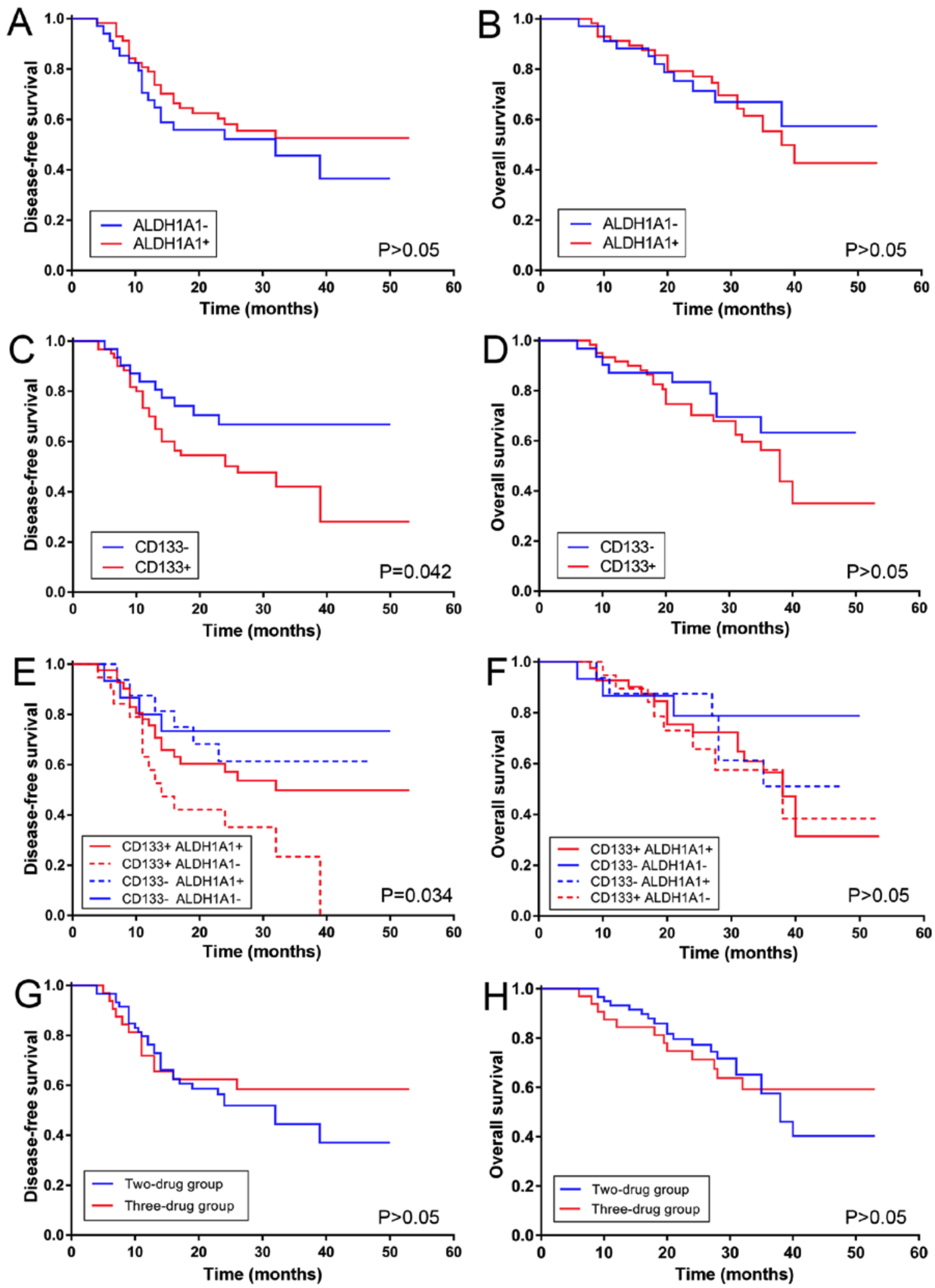

Figure 3. Survival time of patients with gastric cancer. DFS and OS curves. (A) DFS and (B) OS curves for ALDH1A1 expression. (C) DFS and (D) OS curves for CD133 expression. (E) DFS and (F) OS curves for ALDH1A1 and CD133 co-expression. (G) DFS and (H) OS curves for the two- and three-drug groups. DFS, disease-free survival; OS, overall survival; ALDH1A1, aldehyde dehydrogenase-1A1; CD133, cluster of differentiation 133.

Safety of treatment. Table V presents the adverse events reported in 91 patients. Leukopenia was reported with significant differences in the two-drug group when compared with the three-drug group $(\mathrm{P}=0.039)$. In the two-drug group, the reported adverse events of any grade included leukopenia, anemia, gastrointestinal reactions, thrombocytopenia and peripheral neuropathy. In the three-drug group, the reported adverse events of any grade included leukopenia, anemia, thrombocytopenia, liver and kidney toxicity, gastrointestinal reactions, and peripheral neuropathy. The most common 
Table II. Survival rate comparison between the two-drug group and three-drug group.

\begin{tabular}{lcccccc}
\hline Variable & \multicolumn{3}{c}{ Disease-free survival rate (\%) } & \multicolumn{3}{c}{ Overall survival rate (\%) } \\
\hline Time (years) & 1 & 2 & 3 & 1 & 2 & 3 \\
Two drugs & 76.3 & 52.9 & 37.8 & 93.2 & 76.5 & 52.8 \\
Three drugs & 71.9 & 60.0 & 48.0 & 87.5 & 70.0 & 50.0 \\
P-value & 0.645 & 0.537 & 0.426 & 0.357 & 0.521 & 0.833 \\
\hline
\end{tabular}

Table III. Multivariate analysis showing factors associated with disease free survival rate in 91 cases of gastric cancer.

\begin{tabular}{lccccr}
\hline Variable & B & Hazard ratio & Lower & Upper & P-value \\
\hline CD133 & 0.829 & 2.292 & 1.114 & 4.713 & 0.024 \\
Sex & -0.961 & 0.383 & 0.171 & 0.855 & 0.019 \\
Stage (IIIB vs. IIIA) & 1.010 & 2.746 & 1.306 & 5.773 & 0.004 \\
Vascular invasion & 0.627 & 1.872 & 1.003 & 3.494 & 0.049 \\
\hline
\end{tabular}

CD133, cluster of differentiation 133 .

Table IV. Multivariate analysis showing factors associated with overall survival rate in 91 cases of gastric cancer.

\begin{tabular}{lrcccc}
\hline Variable & B & Hazard ratio & Lower & Upper & P-value \\
\hline Sex & -1.216 & 0.296 & 0.112 & 0.786 & 0.015 \\
Tumor size & 0.841 & 2.320 & 1.125 & 4.784 & 0.023 \\
Vascular & 0.923 & 2.516 & 1.227 & 5.162 & 0.012 \\
invasion & & & & & \\
\hline
\end{tabular}

$\mathrm{B}$ value refers to the regression coefficient and the intercept (constant term).

adverse events in the two groups were neutropenia, anemia and gastrointestinal reactions. Adverse events were significantly improved after receiving dose reductions.

\section{Discussion}

Expression of CSC markers have been reported to exist in normal adult stem/progenitor cells as well as in CSCs $(25,26)$, and $\mathrm{ALDH} 1^{+}$cells are mainly located at the normal crypt bottom in the colon and stomach $(15,27)$. The present study examined the expression of two CSC markers (ALDH1A1 and CD133) in the normal and cancerous tissue of the stomach. Positive staining for ALDH1A1 was detected at the crypt bottom of the stomach, especially in parietal cells. Immunoreactivity in the tumors was stronger than in normal tissues; however, staining for CD133 was not detected in the normal stomach tissues.

In the cancerous tissue of the stomach, ALDH1A1 was detected in $62 \%(57 / 91)$ of cases. ALDH1A1 ${ }^{+}$was associated with $\mathrm{T} / \mathrm{N}$ stage and histological type. It has been previously reported that patients with advanced $\mathrm{T} / \mathrm{N}$ stage, as well as undifferentiated have poor survival (20). However, the results of the present study suggest that overexpression of ALDH1A1 is not significantly associated with survival for DFS or OS. The separation of survival curves was observed between $\mathrm{ALDH} 1 \mathrm{~A} 1^{+}$and ALDH1A1 ${ }^{-}$for DFS, in spite of no statistical significance, and ALDH1A1' ${ }^{-}$showed slightly better DFS. It was hypothesized that diverse chemotherapies may have changed the survival of these cases through various mechanisms. In contrast to these results, Levi et al (28) reported that ALDH1 was expressed in very low levels in normal human gastric mucosa, however was significantly increased in gastric adenocarcinomas. Li et al (29) also observed that ALDH1A1 was a poor independent prognostic factor for both OS and recurrence-free survival. However, it has been documented that high ALDH1A1 expression does not always associate with highly malignant phenotypes and poor clinical outcomes in GCs. Wakamatsu et al (30) reported that the expression of ALDH1 had no prognostic impact on the 5-year OS. Li et al (31) used an online database and determined that unlike breast cancer, the mRNA expression of ALDH1A1 in GC was not significantly associated with the OS of patients with GC. ALDH1 is able to convert aldehydes into carboxylic acids in several types of normal tissues such as liver and lungs $(27,28)$. Recently, an increasing body of evidence has revealed that ALDH1, especially ALDH1A1, can modulate cell differentiation, proliferation, survival, and the cellular responses to oxidative stress in CSCs (13). ALDH1A1 has also been shown to mediate epithelial-to-mesenchymal transition, an important phenomenon associated with tumor invasion and metastasis (32). This study suggested that ALDH1A1 was associated with local tumor invasion and lymphatic metastasis; however, the impact on prognosis requires further investigation.

CD133 is a marker that plays an important role in the invasiveness of tumor cells $(11,12)$. In the present study, CD133 was detected in $66 \%(60 / 91)$ of cases. CD133+ was sociated with advanced $\mathrm{T}$ stage and poor DFS in patients with GC, while $\mathrm{N}$ stage, differentiation and poor OS were not associated. CD133 expression could be broadly divided into two types: 
Table V. Adverse events in two drugs and three drugs groups.

\begin{tabular}{|c|c|c|c|c|c|c|c|c|}
\hline \multirow[b]{2}{*}{ Adverse events } & \multicolumn{5}{|c|}{ Grade $(n=91)$} & \multicolumn{3}{|c|}{ Treatment $(\mathrm{n}=91)$} \\
\hline & 0 & 1 & 2 & 3 & 4 & Two drugs & Three drugs & P-value \\
\hline Leukopenia & 46 & 27 & 15 & 3 & 0 & 24 & 21 & $0.039^{\mathrm{a}}$ \\
\hline Anemia & 67 & 19 & 5 & 0 & 0 & 12 & 12 & 0.180 \\
\hline Thrombocytopenia & 83 & 5 & 3 & 0 & 0 & 5 & 3 & 0.972 \\
\hline Gastrointestinal reactions & 76 & 15 & 4 & 0 & 0 & 12 & 7 & 0.307 \\
\hline Peripheral neuropathy & 87 & 3 & 1 & 0 & 0 & 2 & 2 & 0.611 \\
\hline Liver and kidney toxicity & 90 & 1 & 0 & 0 & 0 & 0 & 1 & 0.172 \\
\hline Hand-foot syndrome & 91 & 0 & 0 & 0 & 0 & 0 & 0 & N/A \\
\hline
\end{tabular}

Adverse events were evaluated according to the National Cancer Institute's Common Terminology Criteria for Adverse Events (version 3.0) (23). ${ }^{\mathrm{a}} \mathrm{P}<0.05$.
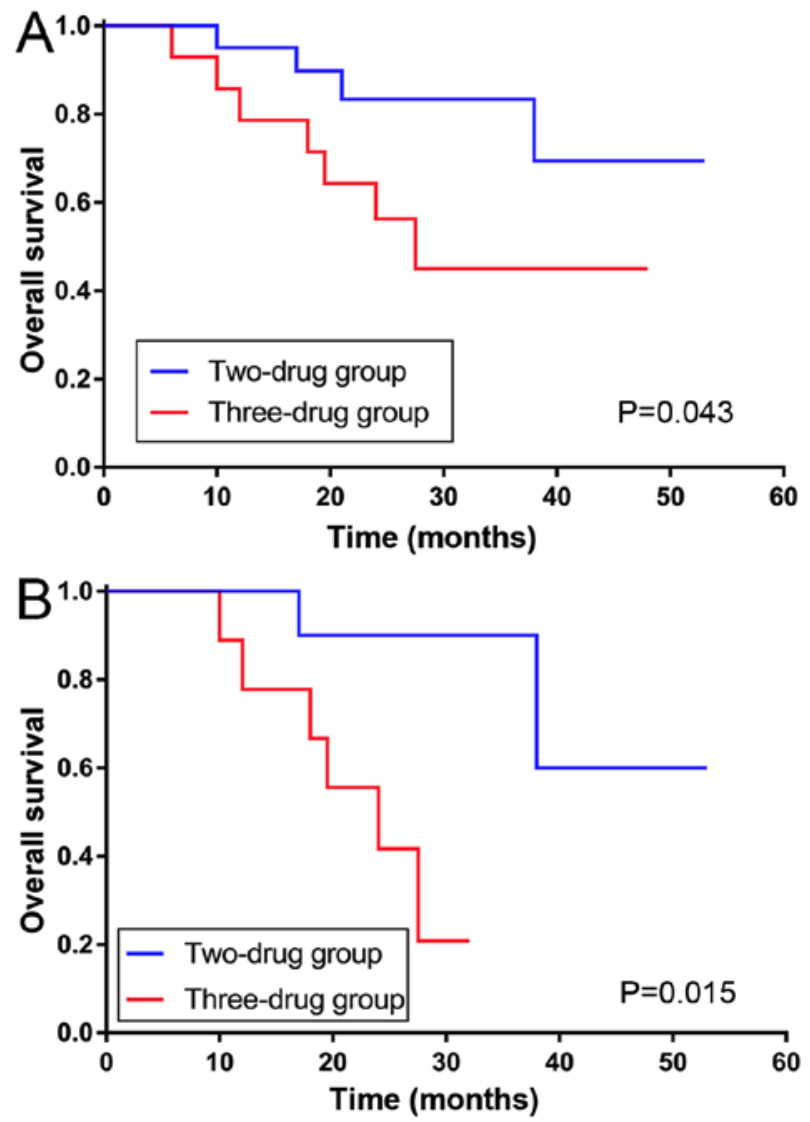

Figure 4. OS curves of subgroups. OS curve for the (A) ALDH1 A1' subgroup, and (B) CD133 ${ }^{+}$and ALDH1 A1 ${ }^{-}$subgroups. OS, overall survival; ALDH1A1, aldehyde dehydrogenase-1 A1; CD133, cluster of differentiation 133 .

Glandular-luminal cell membrane surface expression (luminal expression, L-type) and cytoplasmic expression (C-type) (11). L-type was more common in differentiated GC, while C-type was more common in undifferentiated GC; both expression types were observed in the tissue sections used in the present study. Previous studies demonstrated that overexpression of CD133 was associated with poor prognosis, especially for III/IV stage disease (30,33). Lee et al (34) reported that CD133 was expressed in differentiated GCs and undifferentiated GCs, despite $\mathrm{CD} 133^{+} \mathrm{GCs}$ having significantly poorer prognosis. By contrast, there has also been previous research that has revealed that undifferentiated GC expressed increased levels of CD133 than differentiated GC, and high levels of CD133 expression led to an increase in the malignancy grades of GC (35). Further research is required to elucidate the cause of this difference. These results reveal that positive CD133 expression can predict clinicopathological features and poorer survival in patients with GC. In addition, CD133 may play a critical role in the pathophysiology, integration and complementation of GC. Yu et al (36) demonstrated that inhibition of $C D 133$ gene expression reduced the ability of GC cells to proliferate, invade, form clonal sphere, and induce chemo-resistance, as well as form tumors in nude mice, which further supports the results of the present study. Furthermore, it has been reported that CD133 may inhibit 5-FU-induced apoptosis by regulating the expression of P-gp and the Bcl-2 family mediated by the phosphoinositide 3-kinase/Akt/p70S6K signaling pathway in GC cells (37), which provides further evidence for the drug resistance associated with CD133 expression.

In addition, the co-expression of ALDH1A1 and CD133 was also associated with DFS in the present study. Patients with ALDH1 $1^{-}$and $\mathrm{CD}_{133^{+}}$had poor DFS, and those with ALDH1 A1 ${ }^{-}$and $\mathrm{CD} 133^{-}$had better DFS ( $\left.\mathrm{P}=0.034\right)$. This difference was not observed in OS. A larger sample size is required to examine and conduct further research to verify these differences.

Surgery has become a principle treatment for resectable GC (16). For further treatment, the GASTRIC team (38) conducted a meta-analysis involving 17 randomized, controlled trials over the past few decades. The results verified that adjuvant chemotherapy displayed statistically significant advantages than surgery alone. In addition, the ACTS-GC (17) and CLASSIC (18) trials established fundamental principles of adjuvant chemotherapy. The NCCN Gastric Cancer Clinical Practice Guidelines in Oncology (version 1.2018) (23) suggest that $\mathrm{DCF} / \mathrm{mDCF}$ could be used for locally advanced patients with GC. The aim of the present study was to evaluate the relevance of adding taxane and the survival of patents with stage III disease, and different ALDH1 A1 and CD133 expression levels. 
In general, patients with stage III GC have deeper local invasion, and a large number of cases have lymph node metastasis (20). For these patients, it is necessary to examine whether increasing the intensity of drug treatment benefits the patient's survival. Liu et al (39) published a study that compared whether the three-drug combination was superior to the two-drug combination. Subgroup analysis revealed no significant difference between the two groups. The patients enrolled in the present study were treated with three drugs (DCF or mDCF) or two drugs (XELOX with SOX or mFOLFOX). The results revealed that there was no statistical difference between the two- and three-drug groups in regard to DFS and OS for all 91 patients. The addition of taxane did not induce significant survival benefits to patients.

In regard to subgroup analysis, the ALDH1A1' subgroup exhibited a better response to two-drug therapy. The addition of taxane was shown to induce few benefits for ALDH1A1 ${ }^{+}$. Larzabal et al (40) indicated that in in vivo experiments in lung cancer, paclitaxel, although reducing primary tumor growth, promoted the selection of $\mathrm{ALDH}^{+}$cells that likely modify the lung microenvironment to foster metastasis. Świerczewska et al (41) reported that in the W1 cell line, short-term treatment with PAC upregulated the expression of the ALDHIAl gene. That study hypothesized that by adding taxane to traditional adjuvant chemotherapy, the level of ALDH1A1 may rise, which in turn leads to poorer survival for these patients, and promotes metastatic spread.

The CD133- and CD133+ ${ }^{+}$subgroups did not show any associations with chemotherapies. Patients with $\mathrm{CD}_{133^{+}}$had stronger drug resistance, higher relapse rates and lower 5-year survival rates compared with patients with $\mathrm{CD} 133^{-}$(34). In the present study, it was concluded that $\mathrm{CD} 133^{+}$equated to a poorer DFS, however the addition of taxane in traditional adjuvant chemotherapy did not produce significant benefits for both $\mathrm{CD}_{133}{ }^{-}$and $\mathrm{CD} 133^{+}$expression. Future work should investigate whether CD133 expression was associated with drug resistance, and whether targeting CD133 with specific drugs could be of clinical benefit in patients.

However, the present study has limitations. Further investigation is vital to clarify the role of ALDH1A1 and CD133 in drug resistance of $\mathrm{GC}$, as well as the specific cellular regulatory mechanism of ALDH1A1 and CD133 in the tumorigenesis. In addition, a larger sample of research is required to further prove the association of ALDH1A1 with DFS and OS for patients with GC.

Based on the results of the present study, it was concluded that ALDH1A1 plays an important role in tumor invasion and metastasis. In addition, CD133 may be strongly associated with tumor invasion and prognosis. Furthermore, the taxane-based triple chemotherapeutic regimen produced no benefits for patients, which may help to guide future clinical therapies.

\section{Acknowledgements}

Not applicable.

\section{Funding}

The present study was supported by Major Projects of Natural Science Technology Research of University in Anhui Province (grant no. KJ2018ZD019) and Projects of Anhui Province Natural Science Fund (grant no. 1808085MH306).

\section{Availability of data and materials}

All data generated or analyzed during this study are included in this published article.

\section{Authors' contributions}

WTL and KSG conceived and designed the present study. YYZ, MG and WBL contributed to the design of the present study. WTL and WBL obtained the tumor samples and the clinical data. WTL and MG analyzed the data. KSG and YYZ reviewed and edited the manuscript. All authors read and approved the manuscript and agree to be accountable for all aspects of the research in ensuring that the accuracy or integrity of any part of the work are appropriately investigated and resolved.

\section{Ethics approval and consent to participate}

The present study was approved by the Ethics Committee of The First Affiliated Hospital of Anhui Medical University, and written informed consent was obtained from all patients involved.

\section{Patient consent for publication}

Not applicable.

\section{Competing interests}

The authors declare that they have no competing interests.

\section{References}

1. Torre LA, Bray F, Siegel RL, Ferlay J, Lortet-Tieulent J and Jemal A: Global cancer statistics, 2012. CA Cancer J Clin 65: 87-108, 2015.

2. Reya T, Morrison SJ, Clarke MF and Weissman IL: Stem cells, cancer, and cancer stem cells. Nature 414: 105-111, 2001.

3. Taniguchi $\mathrm{H}$, Moriya C, Igarashi $\mathrm{H}$, Saitoh A, Yamamoto $\mathrm{H}$, Adachi Y and Imai K: Cancer stem cells in human gastrointestinal cancer. Cancer Sci 107: 1556-1562, 2016.

4. Garvalov BK and Acker T: Cancer stem cells: A new framework for the design of tumor therapies. J Mol Med (Berl) 89: 95-107, 2011.

5. Corbeil D, Karbanová J, Fargeas CA and Jászai J: Prominin-1 (CD133): Molecular and cellular features across species. Adv Exp Med Biol 777: 3-24, 2013.

6. Yin AH, Miraglia S, Zanjani ED, Almeida-Porada G, Ogawa M, Leary AG, Olweus J, Kearney J and Buck DW: AC133, a novel marker for human hematopoietic stem and progenitor cells. Blood 90: 5002-5012, 1997

7. Huang R, Mo D, Wu J, Ai H and Lu Y: CD133 expression correlates with clinicopathologic features and poor prognosis of colorectal cancer patients: An updated meta-analysis of 37 studies. Medicine (Baltimore) 97: e10446, 2018.

8. Sukowati CHC: Heterogeneity of hepatic cancer stem cells. Adv Exp Med Biol 1139: 59-81, 2019.

9. Reyes EE, Gillard M, Duggan R, Wroblewski K, Kregel S, Isikbay M, Kach J, Brechka H, Weele DJ, Szmulewitz RZ and Griend DJ: Molecular analysis of CD133-positive circulating tumor cells from patients with metastatic castration-resistant prostate cancer. J Transl Sci 1, 2015.

10. Chen E, Zeng Z, Bai B, Zhu J and Song Z: The prognostic value of CSCs biomarker CD133 in NSCLC: A meta-analysis. Oncotarget 7: 56526-56539, 2016. 
11. Ishigami S, Ueno S, Arigami T, Uchikado Y, Setoyama T, Arima H, Kita Y, Kurahara H, Okumura H, Matsumoto M, et al: Prognostic impact of CD133 expression in gastric carcinoma. Anticancer Res 30: 2453-2457, 2010.

12. Hashimoto K, Aoyagi K, Isobe T, Kouhuji K and Shirouzu K: Expression of CD133 in the cytoplasm is associated with cancer progression and poor prognosis in gastric cancer. Gastric Cancer 17: 97-106, 2014.

13. Tomita H, Tanaka K, Tanaka T and Hara A: Aldehyde dehydrogenase 1A1 in stem cells and cancer. Oncotarget 7: 11018-11032, 2016.

14. Ginestier C, Hur MH, Charafe-Jauffret E, Monville F, Dutcher J, Brown M, Jacquemier J, Viens P, Kleer CG, Liu S, et al: ALDH1 is a marker of normal and malignant human mammary stem cells and a predictor of poor clinical outcome. Cell Stem Cell 1: 555-567, 2007.

15. Huang EH, Hynes MJ, Zhang T, Ginestier C, Dontu G, Appelman H, Fields JZ, Wicha MS and Boman BM: Aldehyde dehydrogenase 1 is a marker for normal and malignant human colonic stem cells (SC) and tracks SC overpopulation during colon tumorigenesis. Cancer Res 69: 3382-3389, 2009.

16. Cardoso R, Coburn NG, Seevaratnam R, Mahar A, Helyer L, Law $\mathrm{C}$ and Singh S: A systematic review of patient surveillance after curative gastrectomy for gastric cancer: A brief review. Gastric Cancer 15 (Suppl 1): S164-S167, 2012.

17. Sakuramoto $S$, Sasako M, Yamaguchi T, Kinoshita $T$, Fujii M, Nashimoto A, Furukawa H, Nakajima T, Ohashi Y, Imamura $\mathrm{H}$, et al: Adjuvant chemotherapy for gastric cancer with S-1, an oral fluoropyrimidine. N Engl J Med 357: 1810-1820, 2007.

18. Bang YJ, Kim YW, Yang HK, Chung HC, Park YK, Lee KH, Lee KW, Kim YH, Noh SI, Cho JY, et al: Adjuvant capecitabine and oxaliplatin for gastric cancer after D2 gastrectomy (CLASSIC): A phase 3 open-label, randomised controlled trial. Lancet 379: 315-321, 2012.

19. Oken MM, Creech RH, Tormey DC, Horton J, Davis TE, McFadden ET and Carbone PP: Toxicity and response criteria of the Eastern Cooperative Oncology Group. Am J Clin Oncol 5 : 649-655, 1982

20. Ilhan E, Ureyen $O$ and Meral UM: Ongoing problems concerning 7th TNM staging system and proposals for 8th TNM staging system of gastric cancer. Prz Gastroenterol 11: 223-225, 2016.

21. Oo AKK, Calle AS, Nair N, Mahmud H, Vaidyanath A, Yamauchi J, Khayrani AC, Du J, Alam MJ, Seno A, et al: Up-regulation of PI 3-kinases and the activation of PI3K-Akt signaling pathway in cancer stem-like cells through DNA hypomethylation mediated by the cancer microenvironment. Trans Oncol 11: 653-663, 2018

22. Jiang Q, Zhang Q, Wang S, Xie S, Fang W, Liu Z, Liu J and Yao K: A fraction of $\mathrm{CD} 133^{+} \mathrm{CNE} 2$ cells is made of giant cancer cells with morphological evidence of asymmetric mitosis. J Cancer 6: 1236-1244, 2015.

23. Network NCCN. (NCCN) Clinical Practice Guidelines in Oncology. Gastric cancer, version 1. 2018. 2018. Available from: https://www.ncen.org/professionals/physician_gls/pdf/gastric.pdf

24. Basch E, Iasonos A, McDonough T, Barz A, Culkin A, Kris MG, Scher HI and Schrag D: Patient versus clinician symptom reporting using the National Cancer Institute Common Terminology criteria for adverse events: Results of a questionnaire-based study. Lancet Oncol 7: 903-909, 2006.

25. Clevers H: The cancer stem cell: Premises, promises and challenges. Nat Med 17: 313-319, 2011.

26. Mills JC and Shivdasani RA: Gastric epithelial stem cells. Gastroenterology 140: 412-424, 2011.

27. Deng S, Yang X, Lassus H, Liang S, Kaur S, Ye Q, Li C, Wang LP, Roby KF, Orsulic S, et al: Distinct expression levels and patterns of stem cell marker, aldehyde dehydrogenase isoform 1 (ALDH1), in human epithelial cancers. PLoS One 5: e10277, 2010.
28. Levi E, Sochacki P, Khoury N, Patel BB and Majumdar AP Cancer stem cells in Helicobacter pylori infection and aging: Implications for gastric carcinogenesis. World J Gastrointest Pathophysiol 5: 366-372, 2014.

29. Li XS, Xu Q, Fu XY and Luo WS: ALDH1A1 overexpression is associated with the progression and prognosis in gastric cancer. BMC cancer 14: 705, 2014.

30. Wakamatsu Y, Sakamoto N, Oo HZ, Naito Y, Uraoka N, Anami K, Sentani K, Oue N and Yasui W: Expression of cancer stem cell markers ALDH1, CD44 and CD133 in primary tumor and lymph node metastasis of gastric cancer. Pathol Int 62: 112-119, 2012.

31. Li K, Guo X, Wang Z, Li X, Bu Y, Bai X, Zheng L and Huang Y: The prognostic roles of ALDH1 isoenzymes in gastric cancer. Onco Targets Ther 9: 3405-3414, 2016.

32. Biddle A, Liang X, Gammon L, Fazil B, Harper LJ, Emich H, Costea DE and Mackenzie IC: Cancer stem cells in squamous cell carcinoma switch between two distinct phenotypes that are preferentially migratory or proliferative. Cancer Res 71 : 5317-5326, 2011.

33. Lu L, Wu M, Sun L, Li W, Fu W, Zhang X and Liu T: Clinicopathological and prognostic significance of cancer stem cell markers CD44 and CD133 in patients with gastric cancer: A comprehensive meta-analysis with 4,729 patients involved. Medicine (Baltimore) 95: e5163, 2016

34. Lee HH, Seo KJ, An CH, Kim JS and Jeon HM: CD133 expression is correlated with chemoresistance and early recurrence of gastric cancer. J Surg Oncol 106: 999-1004, 2012.

35. Jiang Y, He Y, Li H, Li HN, Zhang L, Hu W, Sun YM, Chen FL and Jin XM: Expressions of putative cancer stem cell markers $\mathrm{ABCB} 1, \mathrm{ABCG} 2$, and CD133 are correlated with the degree of differentiation of gastric cancer. Gastric Cancer 15: 440-450, 2012.

36. Yu JW, Wang SL, Wu JG, Lu RQ, Ni XC, Cai C and Jiang BJ: Study on the biological characteristics of CD133(+) cells interfered by RNA interference in gastric cancer. ISRN Gastroenterol 2014: 329519, 2014

37. Zhu Y, Yu J, Wang S, Lu R, Wu J and Jiang B: Overexpression of CD133 enhances chemoresistance to 5-fluorouracil by activating the PI3K/Akt/p70S6K pathway in gastric cancer cells. Oncol Rep 32: 2437-2444, 2014

38. Paoletti X, Oba K, Burzykowski T, Michiels S, Ohashi Y, Pignon JP, Rougier P, Sakamoto J, Sargent D, et al: GASTRIC (Global Advanced/Adjuvant Stomach Tumor Research International Collaboration) Group: Benefit of adjuvant chemotherapy for resectable gastric cancer: A meta-analysis. JAMA 303: 1729-1737, 2010.

39. Liu T, Li W, Sun Y and Shen K: Adjuvant chemotherapy for gastric cancer: Less drug, same efficacy. J Clin Oncol 29: 114, 2011.

40. Larzabal L, El-Nikhely N, Redrado M, Seeger W, Savai R and Calvo A: Differential effects of drugs targeting cancer stem cell (CSC) and non-CSC populations on lung primary tumors and metastasis. PLoS One 8: e79798, 2013.

41. Świerczewska M, Klejewski A, Brązert M, Kaźmierczak D, Iżycki D, Nowicki M, Zabel M and Januchowski R: New and old genes associated with primary and established responses to paclitaxel treatment in ovarian cancer cell lines. Molecules 23: E891, 2018 .

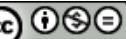

This work is licensed under a Creative Commons Attribution-NonCommercial-NoDerivatives 4.0 International (CC BY-NC-ND 4.0) License. 